\title{
Solution of Optimal Power Flow Problem Incorporating Various FACTS Devices
}

\author{
Nanda Kumar Easwaramoorthy \\ Asst. Professor / Dept. of E.E.E., \\ Sri Krishna College of Technology, \\ Kovaipudur, Coimbatore.
}

\author{
R. Dhanasekaran, PhD. \\ Director- Research, SyedAmmal \\ Engineering College, Ramnad.
}

\begin{abstract}
This research paper presents a new approach for placing the optimal location of FACTS controllers in a multi machine power system using mat lab coding. Using the proposed method, the location of FACTS controller, their type and rated values are optimized simultaneously. Among the various FACTS controllers, TCSC and UPFC are considered. OPF is one of the most important processes in power system, which improves the system performance by satisfying certain constraints.

Generally, different optimization methods are used in the literature to solve the OPF problem. In some research works, the optimization process is done by considering total fuel cost or by considering the environmental pollution that occurs during power generation. But in some other research works, FACTS controllers are used to improve the power flow without considering the power generation cost.

The OPF problem is one of the most extensively studied topics in the power system community. In power system operation, $\mathrm{OPF}$ is an extended problem of $\mathrm{ED}$ which considers several parameters such as generator voltage, transformer tap change, SVC, and includes constraints such as transmission line and transformer loading limits, bus voltage limit, and stability margin limit. The main function of OPF is to select the optimal operation state of a power system, in the time of meeting some particular constraints. OPF study plays a key role in the EMS, where the entire operation of the system is regulated in each possible real time intervals.
\end{abstract}

\section{Keywords}

OPF, TCSC, UPFC, ED, EMS.

\section{INTRODUCTION}

This paper proposes an OPF problem which is realized by means of Particle Swarm Optimization algorithm. Particle Swarm Optimization (PSO) is a population based stochastic optimization technique developed by Dr. Eberhart and Dr. Kennedy in 1995, inspired by social behavior of bird flocking or fish schooling. PSO optimizes a problem by having a population of candidate solutions, here dubbed particles, and moving these particles around in the search-space according to simple mathematical formulae over the particle's position and velocity.

The equality constraints are the nodal power balance equations, whereas the inequality constraints are the limits of all control or state variables. The physical laws controlling the power generation of transmission systems and the operating limitations of the equipment are the constraints involved for optimizing the objective function. OPF is the evaluation of the best settings of the control variables such as the Active Power and Voltages of Generators, Discrete variables like Transformer taps, Continuous variables like the Shunt reactors and Capacitors, and other continuous and discrete variables, in order to achieve a common objective such as reduction of operating cost or Social Welfare while respecting all the system limits for secure operation.

The possibility of operating power systems at the lower cost, while satisfying the given transmission and security constraints is one of the main current issues in elongating the transmission capacity through the use of FACTS devices. FACTS devices can direct the active and reactive power control and flexible to voltage-magnitude control simultaneously, because of their adaptability and fast control characteristics. With the aid of FACTS technology, namely SVC, STATCOM, SSSC and UPFC etc., the bus voltages, line impedances and phase angles in the power system can be controlled quickly and flexibly.

The equality constraints are the nodal power balance equations, whereas the inequality constraints are the limits of all control or state variables [4]. The physical laws controlling the power generation of transmission systems and the operating limitations of the equipment are the constraints involved for optimizing the objective function [9]. OPF is the evaluation of the best settings of the control variables such as the Active Power and Voltages of Generators, Discrete variables like Transformer taps, Continuous variables like the Shunt reactors and Capacitors, and other continuous and discrete variables, in order to achieve a common objective such as reduction of operating cost or Social Welfare while respecting all the system limits for secure operation [5].

The solution techniques used for the OPF problem are linear programming, quadratic programming, gradient techniques, interior point techniques and stochastic optimization models [8]. These techniques depend on convexity to achieve the global optimum solution, and such that they are forced to simplify the relationships to ensure convexity [11]. Furthermore, to solve the OPF problems, several heuristic algorithms such as EP, TS, TS/SA, ITS and IEP have been proposed [9].

\section{FACTS DEVICES TO BE INCORPORATED TO OPF PROBLEM}

\subsection{TCSC}

The TCSC can serve as the capacitive or inductive compensation respectively by modifying the reactance of the transmission line. In this paper, the reactance of the transmission line is adjusted by TCSC directly. The rated value of TCSC is a function of the reactance of the transmission line where the TCSC is located. 


$$
X i_{j}=X_{\text {Line }}+X_{T C S C}
$$

$$
X_{\text {TCSC }}=r_{t \mathrm{csc}} . X_{\text {line }}
$$

where $X_{\text {Line }}$ is the reactance of the transmission line and $r_{t c s c}$ is the coefficient which represents the compensation degree of TCSC. To avoid over compensation, the working range of the TCSC is between $0.7 \mathrm{X}_{\mathrm{Line}}$ and $0.2 \mathrm{X}_{\mathrm{Line}}$.

\subsection{UPFC}

The UPFC is a combination of shunt and series controller. It has three controllable parameters namely, the magnitude of the boosting injected voltage (UT), phase of this voltage (ØТ) and the exciting transformer reactive current (Iq).

\section{RELATIVE MERITS OF FACTS}

\subsection{Balancing of load flows}

This enables the load flow on parallel circuits and different voltage levels to be optimized, with a minimum of power wheeling, the best possible utilization of the lines, and a minimizing of overall system losses at the same time.

3.2 Increasing of first swing stability, power oscillation damping, and voltage stability

This enables a maximizing of system availability as well as of power transmission capability over existing as well as new lines. Thus, more power can be transmitted over fewer lines, with a saving of money as well as of environmental impact of the transmission link.

\subsection{Mitigation of sub synchronous resonance risk}

Sub synchronous resonance (SSR) is a phenomenon which can be associated with series compensation under certain adverse conditions. The elimination of the risk of SSR even for the most onerous conditions means that the series compensation concept can be utilized in situations where it would otherwise not have been undertaken, thereby widening the usefulness of series compensation.

\subsection{Power system interconnection}

Interconnecting of power systems is becoming increasingly widespread as part of power exchange between countries as well as regions within countries in many parts of the world. Such are found in the Nordic countries, Argentina, and Brazil.

\section{IMPROVEMENTS IN POWER SYSTEM}

\section{Stability}

The cost of losing synchronous operation through a transient instability is extremely high in modern power systems. Consequently, utility engineers often perform a large number of stability studies in order to avoid the problem. Since different operating points of a power system have different stability characteristics, stability can be maintained by searching for one point that respects appropriate stability limits. In the past three decades, power system stabilizers (PSSs) have been extensively used to increase the system damping for low frequency oscillations. However, there have been problems experienced with PSSs over the years of operation. Some of these were due to the limited capability of PSS, in damping only local and not inter area modes of oscillations. In addition, PSSs can cause great variations in the voltage profile under severe disturbances and they may even result in leading power factor operation and losing system stability. FACTS have gained a great interest during the last few years, due to recent advances in power electronics.

\section{LOAD FLOW CALCULATIONS}

The load flow calculation is important to compute the power flow between the buses. In our method Newton Raphson method is used for load flow calculation. Newton Raphson method is commonly used technique for load flow calculation. The real and reactive power in each bus is computed using equation $3 \& 4$.

$$
P_{i}=\sum_{k=1}^{N} V_{i} * V_{k}\left(G_{i k} * \cos \theta_{i k}+B_{i k} * \sin \theta_{i k}\right)
$$

$$
Q_{i}=\sum_{k=1}^{N} V_{i} * V_{k}\left(G_{i k} * \sin \theta_{i k}-B_{i k} * \cos \theta_{i k}\right)
$$

where, $N$ is the total number of buses, $V_{i} \& V_{k}$ are the voltage at $i \& k$ bus respectively, $\theta_{i k}$ is the angle between $i \& k$ bus, $G_{i k} \& B_{i k}$ are the conductance and susceptance value respectively.

After computing the power flow between the lines, the amount of power to be generated for the corresponding load with low cost is identified using PSO. In our method, there are two stages of PSO and a neural network is used. Here, PSO is used for generating training dataset to train the neural network. In the first stage, the amount of power generated by each generator for a particular load is computed using PSO and in the second stage, the bus where the FACTS controller is to be connected is identified and using this data, the neural network is trained. From the output of neural network, the amount of power to be generated by each generator for the given load and the location of FACTS controller to be connected are obtained.

\subsection{Computation of Power to be generated for $P_{G i}$}

The amount of power to be generated by each generator is estimated using PSO. The process that takes place in PSO is generation of initial particle, evaluation function and updating the particles. The first step is generating the initial particle by PSO.

\subsection{Generating Initial Particle}

First the total number of generators connected in the system is identified and then the amount of power generated by each generator is calculated by satisfying two constraints. The initial particles to be generated by using PSO are $\left\{P_{G 1}, P_{G 2}, \ldots \ldots . P_{G D}\right\}$. The two constraints that must be satisfied for generating the particle are given below. 
Constraint1: $\sum_{i=1}^{D} P_{G i}=P_{d}+P_{l}$

where, $P_{G i}$ is the total power generated, $P_{d}$ is the total power demand, $P_{l}$ is the total power loss, $D$ is the total number of generator.

Constraint2: $P_{G i}^{\min } \leq P_{G i} \leq P_{G i}^{\max }$

where, $P_{G i}^{\text {min }}$ and $P_{G i}^{\max }$ is the minimum and maximum real power to be generated by $i^{\text {th }}$ generator.

The initial particles are generated by satisfying the above two constraints and after generating the initial particle, the next step is evaluation function.

\subsection{Evaluation Function}

The evaluation function is used to evaluate the initial particle generated in the above step. Here, the cost function is taken as the evaluation function.

Evaluation function,

$$
\begin{gathered}
C=F+\boldsymbol{E} \\
\text { where, FuelCost } F=\sum_{i=1}^{D}\left(a_{i}+b_{i} * P_{G i}+c_{i} * P_{G i}^{2}\right)
\end{gathered}
$$

$$
\text { Emissioncost, } \quad E=\sum_{i=1}^{D}\left(\alpha_{i} * P_{G i}{ }^{2}+\beta_{i} * P_{G i}+\gamma_{i}\right)
$$

where, $a_{i}, b_{i}$ and $c_{i}$ are the cost coefficients of the $i^{t h}$ generator, $P_{G i}$ is the real power of the $i^{\text {th }}$ generator, and $\alpha_{i}, \beta_{i}$ and $\gamma_{i}$ are the coefficients of the $i^{t h}$ generator emission characteristics.

\subsection{Updating Initial Particles}

Updating the particles is an important process in PSO. In this stage, the initial particles generated are updated and then the fitness values are calculated. The particles are updated using the equation are given below $v[]=v[]+c 1 * \operatorname{rand}() *(\operatorname{pbest}[]-\operatorname{present}[])+c 2 * \operatorname{rand}() *($ gbest []$-\operatorname{present}[])$

(10)

$$
\operatorname{present}[]=\operatorname{present}[]+v[]
$$

$v[]$ is the particle velocity, present [] is the current particle, pbest [ ] and gbest [ ] are best fitness value and best value from any particle in the population respectively, $\operatorname{rand}()$ is the random number between $(0,1)$ and $c 1, c 2$ are learning factors.

By using the above equation, initial particles are updated and a new particle is generated. The total number of new particles is generated based on the number of iterations applied. Then, the evaluation function is applied to the newly generated particles and the particle with low cost is selected as the best particle.

Repeat the above process by randomly generating new set of generator values and the process are repeated for $n$ times, so that $n$ set of data is generated. The following equation gives the procedure to obtain $n$ set of data generated from PSO

$$
S=\left[\begin{array}{lll}
P_{G 11} & P_{G 12} \ldots \ldots . P_{G 1 D} \\
P_{G 21} & P_{G 22} \ldots \ldots P_{G 2 D} \\
\cdot & \\
\cdot & \\
P_{G n 1} & P_{G n 2} \ldots \ldots P_{G n D}
\end{array}\right]\left[\begin{array}{l}
C_{1} \\
C_{2} \\
\cdot \\
\cdot \\
C_{n}
\end{array}\right]
$$

From the above generated data, the minimum cost function is taken as the power generated by the generator with low cost.

\section{Optimal Power Flow with FACTS Controllers}

The formulation of the optimal allocation of FACTS
controllers can be expressed as Minimize
$C_{\text {Total }}=C_{1}(f)+C_{2}(P G)$

$$
\begin{aligned}
& \text { Subjected to } E(f, g)=0 \\
& B_{1}(f)\left\langle b_{1}, B_{2}(g)\left\langle b_{2}\right.\right.
\end{aligned}
$$

Where

$\mathrm{C}_{\text {Total }}$ : the overall cost objective function which includes the average investment costs of FACTS devices $\mathrm{C}_{1}$ (f) and the generation $\operatorname{cost} \mathrm{C}_{2}(\mathrm{PG})$.

E (f.g) : the conventional power flow equations.

$\mathrm{B}_{1}$ (f) and $\mathrm{B}_{2}(\mathrm{~g})$ are the inequality constraints for FACTS controllers and the conventional power flow respectively.

$\mathrm{f}$ and PG are vectors that represent the variables of FACTS controllers and the active power outputs of the generators.

$\mathrm{g}$ represents the operating state of the power

System.

The unit for generation cost is US\$/Hour and for the investment cost of FACTS controllers are US\$. They must be unified into US\$/Hour. Normally the FACTS controllers will be in service for many years. However only a part of its life time is employed to regulate the power flow. In this paper three years is employed to evaluate the cost function. Therefore the average value of the investment costs are calculated as follows

$$
C_{1}(f)=C(f) /\{8760 X 3\}
$$


As mentioned above, power system parameters can be changed using FACTS controllers. These different parameters derive different results on the objective function. Also, the variation of FACTS locations and FACTS types has also influences on the objective function. Therefore, using the conventional optimization methods are not easy to find the optimal location of FACTS devices, types and control parameters simultaneously.

\section{Optimized Settings of FACTS Devices}

In this paper UPFC is modeled as combination of a TCSC in series with the line and SVC connected across the corresponding buses between which the line is connected. After fixing the location, to determine the best possible settings of FACTS devices for all possible single and multiple contingencies, the optimization problem will have to be solved using Fuzzy Controlled FACTS controller technique.

The objective function for this work is,

Objective $=$ minimize $\{$ SOL and IC $\}$

$S O L=\sum_{C=1}^{M} \sum_{k=1}^{n} a_{k}\left(P_{k} / P_{k^{\max }}\right)^{4}$

where,

$\mathrm{m}$ - Number of single contingency considered

n- Number of lines

ak- weight factor $=1$.

$\mathrm{P}_{\mathrm{k}}$ - real power transfer on branch $\mathrm{k}$.

$\mathbf{P}_{\mathbf{k}}{ }^{\mathbf{m a x}}$ - maximum real power transfer on branch $\mathrm{k}$.

IC - Installation cost of FACTS device

$S O L$ - Represents the severity of overloading

$$
\begin{aligned}
& C_{T C S C}=0.0015 S^{2}-0.71 S+153.75(U S \$ / K V A R) \\
& C_{U P F C}=0.0003 S^{2}-0.2691 S+188.22(U S \$ / K V A R)
\end{aligned}
$$

Where, S - Operating range of UPFC in MVAR

$$
S=\left|Q_{2}-Q_{1}\right|
$$

Q1 - MVAR flow through the branch before placing FACTS device.

Q2 - MVAR flow through branch after placing FACTS device.

\section{Fuzzy Controller and its operation}

The collection of rules is called a rule base. The rules are in the familiar if-then format, and formally the ifside is called the condition and the then-side is called the conclusion (more often, perhaps, the pair is called antecedent - consequent or premise - Conclusion).

A preprocessor, the first block in the structure conditions the measurements before they enter the controller. The first block inside the controller is fuzzification, which converts each piece of input data to degrees of membership by lookup in one or several membership functions. The rules may use several variables both in the condition and exclusion of the rules. The controllers can therefore be applied to both multi-input-multi-output (MIMO) problems and single-inputsingle-output (SISO) problems.

\section{OPF with FACTS Controller using Simulation}

Optimal power flow is one of the important methods used to increase the power flow between the buses. OPF is not only to increase the power flow in the system, but also to generate power based on the requirement with low cost. The power flow between the buses can also be increased by connecting FACTS controller in suitable places. By considering the above problems, here a new method for OPF with FACTS controller using Mat Lab Simulation was proposed. Initially, the load flow between the buses is calculated using Newton raphson method and then the amount of power to be generated by each generator is computed using PSO. Finally, the FACTS controller is placed in a suitable location using PSO and Fuzzy Controller to increase the power flow between the buses. The process that takes place in the proposed method is explained briefly in the below sections.

\section{Identifying UPFC connecting bus}

In the testing stage, if a bus number except the slack bus given as input, it checks the lines which are connected in that bus and based on the reduce in cost and increase in power flow, the next bus where the UPFC is to be connected and the corresponding voltage and angle to be injected in that bus are obtained as output by the neural network.

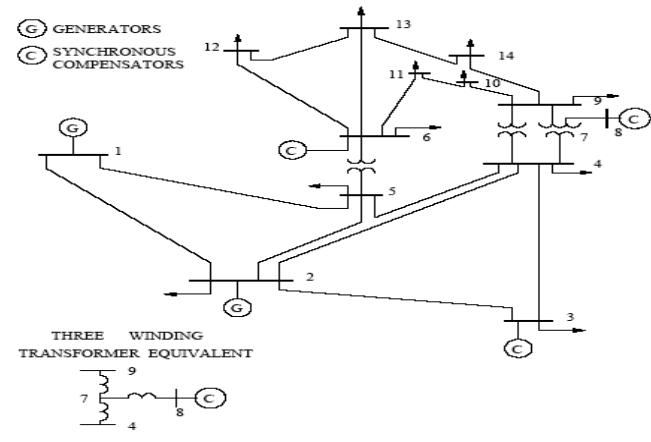

Fig. 1. IEEE standard 14 bus system

In the test system, bus 1 is considered as the slack bus and the base MVA of the system is 100. Bus 2, 13, 22, 23 and 27 are generator bus and all other buses are load bus.

By injecting the voltage and angle value to the line that are identified by the network, and using the amount of power

generated by each generator that are obtained as an output from the first stage of PSO, the power

The proposed technique was implemented in the working platform of 7.11 and tested using IEEE $14 \& 30$ bus systems. 


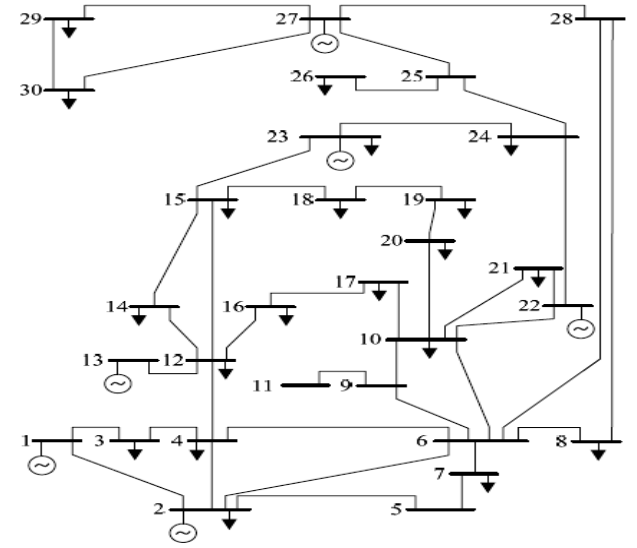

Fig. 2. IEEE standard 30 bus system

In the test system, bus 1 is considered as the slack bus and the base MVA of the system is 100. Bus 2, 13, 22, 23 and 27 are generator bus and all other buses are load bus.

\section{RESULTS AND DISCUSSIONS}

\begin{tabular}{|c|c|c|}
\hline $\begin{array}{c}\text { Objective } \\
\text { function to be } \\
\text { optimized }\end{array}$ & $\begin{array}{l}\text { Suitable } \\
\operatorname{method}(s)\end{array}$ & $\begin{array}{l}\text { Reason to use } \\
\text { those methods }\end{array}$ \\
\hline $\begin{array}{l}\text { Economic } \\
\text { dispatch }\end{array}$ & LP, NR & Fast methods \\
\hline $\begin{array}{l}\text { Economic } \\
\text { dispatch with } \\
\text { non-smooth } \\
\qquad \text { cost } \\
\text { function }\end{array}$ & AI & $\begin{array}{l}\text { Non-linear } \\
\text { problem }\end{array}$ \\
\hline $\begin{array}{l}\text { Economic - } \\
\text { Emission } \\
\text { dispatch }\end{array}$ & Fuzzy & $\begin{array}{l}\text { Suitable for } \\
\text { conflicting } \\
\text { objectives }\end{array}$ \\
\hline $\begin{array}{l}\text { Reactive power } \\
\text { optimization }\end{array}$ & $\begin{array}{c}\text { NLP, } \\
\text { QP,IP,AI }\end{array}$ & Accurate methods \\
\hline $\begin{array}{l}\text { Optimal } \\
\text { location of } \\
\text { FACTS device }\end{array}$ & AI & $\begin{array}{l}\text { Multiobjective } \\
\text { Nonlinear } \\
\text { problem }\end{array}$ \\
\hline Social welfare & $\mathrm{QP}, \mathrm{AI}$ & $\begin{array}{l}\text { Multiobjective } \\
\text { Nonlinear } \\
\text { problem }\end{array}$ \\
\hline $\begin{array}{l}\text { Congestion } \\
\text { management }\end{array}$ & AI & $\begin{array}{l}\text { Multiobjective } \\
\text { Nonlinear } \\
\text { problem }\end{array}$ \\
\hline
\end{tabular}

\begin{tabular}{|l|l|l|}
\hline Security & NLP, IP & $\begin{array}{l}\text { Stable } \\
\text { convergence } \\
\text { OPF }\end{array}$ \\
\hline
\end{tabular}

Table 1. Comparison of various OPF methods

\begin{tabular}{|c|c|c|}
\hline $\begin{array}{c}\text { Generator } \\
\text { bus }\end{array}$ & $\begin{array}{c}\text { Minimum } \\
\text { (MW) }\end{array}$ & $\begin{array}{c}\text { Maximum } \\
\text { (MW) }\end{array}$ \\
\hline 1 & 50 & 200 \\
\hline 2 & 20 & 80 \\
\hline 3 & 15 & 50 \\
\hline 4 & 10 & 35 \\
\hline 5 & 10 & 30 \\
\hline 6 & 12 & 40 \\
\hline
\end{tabular}

Table 1.Generator operating limit

\begin{tabular}{|c|c|}
\hline $\begin{array}{c}\text { Generator } \\
\text { bus }\end{array}$ & $\begin{array}{c}\text { Power generated in each } \\
\text { generator using our } \\
\text { proposed method } \\
\text { (MW) }\end{array}$ \\
\hline 1 & 175.3339 \\
\hline 2 & 56.27446 \\
\hline 3 & 18.98122 \\
\hline 4 & 17.07911 \\
\hline 5 & 13.10195 \\
\hline 6 & 12.22937 \\
\hline
\end{tabular}

Table 2. Power Generated in each generator

\begin{tabular}{|c|c|}
\hline SSSC connected at & Total power loss (MW) \\
\hline $2-5$ & 8.67 \\
\hline $3-4$ & 9.62 \\
\hline $2-4$ & 9.718 \\
\hline $5-7$ & 9.585 \\
\hline $8-28$ & 9.476 \\
\hline
\end{tabular}




\begin{tabular}{|c|c|}
\hline $9-10$ & 9.93 \\
\hline $10-17$ & 9.71 \\
\hline $14-15$ & 9.7452 \\
\hline $12-15$ & 9.9079 \\
\hline $19-20$ & 9.46 \\
\hline $21-23$ & 9.68 \\
\hline $24-25$ & 9.57 \\
\hline
\end{tabular}

Table 3. Total power loss with SSSC

\subsection{Open loop control with SVC}

The following waveforms shows the simulation results for open loop control with SVC

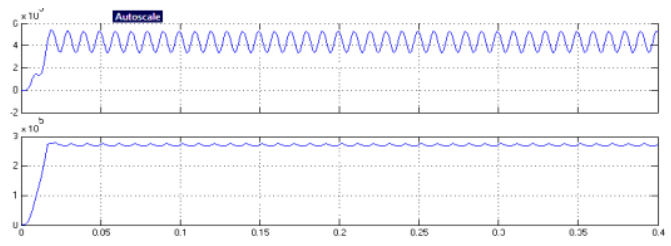

Fig. 3. Open loop control with SVC waveform

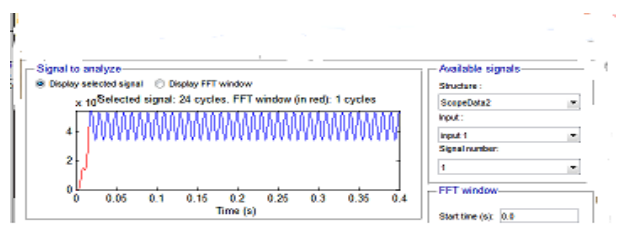

Fig. 4. Open loop control with SVC LT view parameters

\subsection{Closed loop control with TCVR}

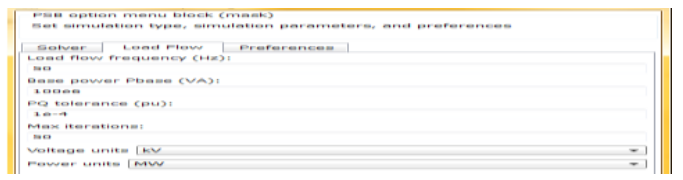

Fig. 5. Open loop control with parameters

\subsection{Closed loop control with SVC}

The following waveforms shows the simulation results for closed loop control with SVC

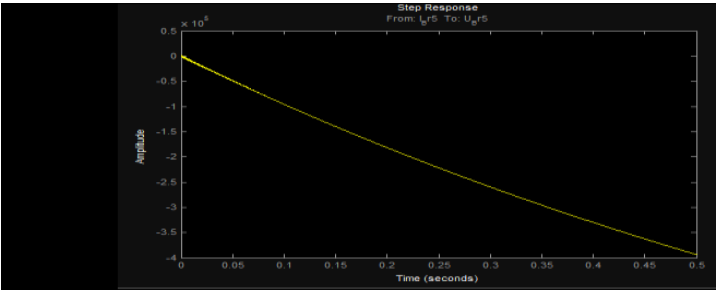

Fig. 6. Closed loop control with SVC waveform

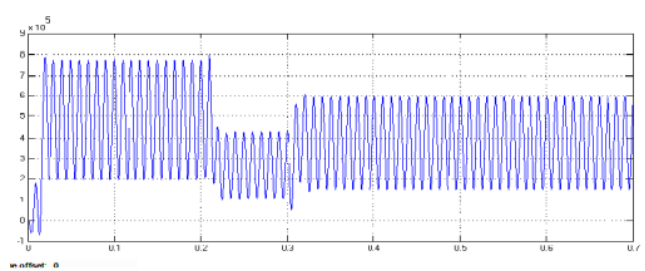

Fig.7. Closed loop control with SVC LT view

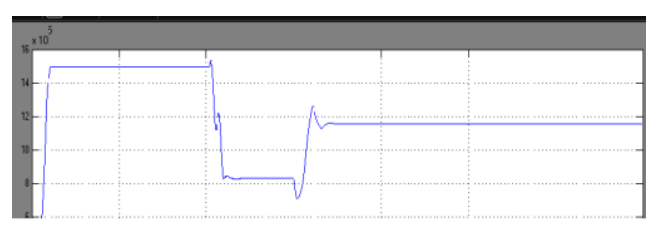

Fig. 8. Closed loop control with TCVR waveform

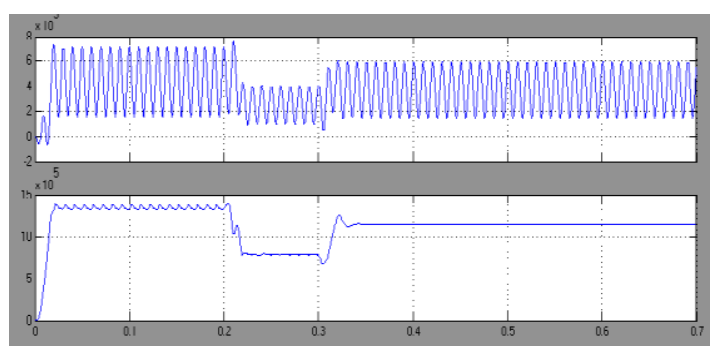

Fig.9. Closed loop control with TCVR

\section{LT view waveform}

\section{CONCLUSION \& FUTURE SCOPE}

In this paper, the proposed method was tested for IEEE $14 \& 30$ bus systems and FACTS controller used in our method is open and closed loops SVC and TCVR. From the above results it is clear that our method has reduced the power losses as well as the total cost in the system. This method to be tested for IEEE 50 bus systems also in future. Also various FACTS controllers like STATCOM, SSSC and UPFC etc., also to be incorporated likely.

\section{REFERENCES}

[1] MithunBhaskar M, SrinivasMuthyala and SyduluMaheswarapu, "Security Constraint Optimal Power Flow (SCOPF) - A Comprehensive Survey", International Journal of Computer Applications, Vol. 11, No.6, pp. 42-52, Dec 2010. 
[2] K. Mani Chandy, Steven H. Low, UfukTopcu and HuanXu, "A Simple Optimal Power Flow Model with Energy Storage", In Proceedings of IEEE Conference on Decision and Control, Atlanta, pp. 1051-1057, Dec 2010.

[3] BrahimGasboui and BoumedieneAllaoua, "Ant Colony Optimization Applied on Combinatorial Problem for Optimal Power Flow Solution", Leonardo Journal of Sciences, Issue. 14, pp. 1-17, June 2009.

[4] Zwe-Lee Gaing; Rung-Fang Chang, "Securityconstrained optimal power flow by mixed-integer genetic algorithm with arithmetic operators", In Proceedings of IEEE Power Engineering Society General Meeting, pp. 1-8, Montreal, 2006.

[5] Mithun Bhaskar M, Srinivas Muthyala and Sydulu Maheswarapu, "Security Constraint Optimal Power Flow (SCOPF) - A Comprehensive Survey", International Journal of Computer Applications, Vol. 11, No.6, pp. 4252, Dec 2010.

[6] N. G. Hingorani and L. Gyugyi, Understanding FACTS concepts and technology of flexible AC transmission systems. New York: IEEE Press, 2000.

[7] R. M. Mathur and R. K. Varma, Thyristor-based FACTS controllers for electrical transmission systems. Piscataway: IEEE Press, 2002.

[8] Keerati Chayakulkheeree and Weerakorn Ongsakul, "Optimal Power Flow Considering Non-Linear Fuzzy Network and Generator Ramprate Constrained", International Energy Journal, Vol. 8, pp.131-138, 2007.

[9] C. Thitithamrongchai and B. Eua-Arporn, "Self-adaptive Differential Evolution Based Optimal Power Flow for Units with Non-smooth Fuel Cost Functions", Journal of Electrical Systems, Vol. 3, No. 2, pp. 88-99, 2007.

[10] J. Carpentier and A. Merlin, "Optimization methods in planning and operation," International Journal of Electrical Power and Energy Systems, vol. 4, pp. 11-18, 1982

[11] K. S. Swarup, "Swarm intelligence approach to the solution of optimal power flow", J. Indian Inst. Sci., Vol.86, pp. 439-455, Oct 2006.

[12] H. Ambriz-Perez, E. Acha, and C. R. Fuerte-Esquivel, "Advanced SVC models for Newton-Raphson load flow and Newton optimal power flow studies," Power Systems, IEEE Transactions on, vol. 15, pp. 129-136, 2000.

[13] T. Orfanogianni, "A flexible software environment for steady-state power flow optimization with series FACTS devices." Diss. ETH Zurich, 2000, pp. 135 S.

[14] TarekBouktir and Linda Slimani, "Optimal Power Flow of the Algerian Electrical Network using an Ant Colony Optimization Method", Leonardo Journal of Sciences, Issue. 7, pp. 43-57, Dec 2005.

[15] TarekBouktir and Linda Slimani, "A Genetic Algorithm for Solving the Optimal Power Flow Problem", Leonardo Journal of Sciences, Issue. 4, pp. 44-58, June 2004.

[16] Mithun M. Bhaskar, SrinivasMuthyala and MaheswarapuSydulu, "A Novel Progressively Swarmed Mixed Integer Genetic Algorithm for Security Constrained Optimal Power Flow (SCOPF)",
International Journal of Engineering, Science and Technology, Vol. 2, No. 11, pp. 34-40, 2010.

[17] KeeratiChayakulkheeree and Weerakorn Ongsakul, "Optimal Power Flow Considering Non-Linear Fuzzy Network and Generator Ramprate Constrained", International Energy Journal, Vol. 8, pp.131-138, 2007.

[18] C. Thitithamrongchai and B. Eua-Arporn, "Self-adaptive Differential Evolution Based Optimal Power Flow for Units with Non-smooth Fuel Cost Functions", Journal of Electrical Systems, Vol. 3, No. 2, pp. 88-99, 2007.

[19] P. K.Roy, S. P. Ghoshal and S.S. Thakur, "Biogeography Based Optimization Approach for Optimal Power Flow Problem Considering Valve Loading Effects", International J. of Recent Trends in Engineering and Technology, Vol. 3, No. 3, pp. 177-181, May 2010.

[20] S. Jaganathan, S. Palanisamy K. Senthilkumaravel and B. Rajesh, "Application of Multi-Objective Technique to Incorporate UPFC in Optimal Power Flow using Modified Bacterial Foraging Technique", International Journal of Computer Applications, Vol.13, No.2, pp. 1824, Jan 2011.

[21] K. S. Swarup, "Swarm intelligence approach to the solution of optimal power flow", J. Indian Inst. Sci., Vol.86, pp. 439-455, Oct 2006.

[22] Mithun M. Bhaskar and SyduluMaheswarapu, "A Hybrid Genetic Algorithm Approach for Optimal Power Flow", TELKOMNIKA, Vol. 9, No. 1, pp. 209-214, April 2011.

[23] Keerati Chayakulkheeree and Weerakorn Ongsakul, "Multi-Objective Optimal Power Flow Considering System Emissions and Fuzzy Constraints", GMSARN International Journal Vol. 1, pp. 1 - 6, 2008.

\section{Appendix}

List of Abbreviations used

OPF - Optimal Power Flow

PSO - Particle Swarm Optimization

EP - Evolutionary Programming

STATCOM - Static Synchronous Compensator

SSSC - Static Synchronous Series Compensator

TS - Tabu Search

TS/SA - hybrid Tabu Search and Simulated Annealing

SA- Simulated Annealing

ITS,- Improved Tabu Search

IEP - Improved Evolutionary Programming

ED - Economic Dispatch

EMS - Energy Management Systems

IEP - Integer Evolutionary Programming

TCSC - Thyristor Controlled Switched Capacitor

TCVR - Thyristor Controlled Variable Reactor

FACTS - Flexible A.C. Transmission Systems

SVC - Static Var Compensator

UPFC - Unified Power Flow Controller 